\title{
Age-Related Differences in The Survival Benefit of Anticoagulants in Sepsis: A Retrospective Sub- Analysis of A Prospective Multicenter study
}

Takeshi Wada ( $\square$ twada1@med.hokudai.ac.jp )

Hokkaido University Faculty of Medicine

Kazuma Yamakawa

Osaka Medical and Pharmaceutical University

Daijiro Kabata

Osaka City University Graduate School of Medicine

Toshikazu Abe

Tsukuba Memorial Hospital

Hiroshi Ogura

Osaka University Graduate School of Medicine

Atsushi Shiraishi

Kameda Medical Center

Daizoh Saitoh

National Defense Medical College

Shigeki Kushimoto

Tohoku University Graduate School of Medicine

Seitaro Fujishima

Keio University School of Medicine

Toshihiko Mayumi

University of Occupational and Environmental Health

Toru Hifumi

St. Luke's International Hospital

Yasukazu Shiino

Kawasaki Medical School

\section{Taka-aki Nakada}

Chiba University Graduate School of Medicine

\section{Takehiko Tarui}

Kyorin University Faculty of Health Sciences

\section{Yasuhiro Otomo}

Tokyo Medical and Dental University

Kohji Okamoto 
Kitakyushu City Yahata Hospital

\section{Yutaka Umemura}

Osaka General Medical Center

\section{Joji Kotani}

Kobe University Graduate School of Medicine

\section{Yuichiro Sakamoto}

Saga University Hospital

\section{Junichi Sasaki}

Keio University School of Medicine

\section{Shin-ichiro Shiraishi}

Aizu Chuo Hospital

\section{Kiyotsugu Takuma}

Kawasaki Municipal Hospital

\section{Ryosuke Tsuruta}

Yamaguchi University Hospital

\section{Akiyoshi Hagiwara}

Center Hospital of the National Center for Global Health and Medicine

\section{Tomohiko Masuno}

Nippon Medical School

\section{Naoshi Takeyama}

Aichi Medical University Hospital

\section{Norio Yamashita}

Kurume University

\section{Hiroto Ikeda}

Teikyo University School of Medicine

\section{Masashi Ueyama \\ Chukyo Hospital}

\section{Satoshi Fujimi}

Osaka General Medical Center

\section{Satoshi Gando}

Sapporo Higashi Tokushukai Hospital

\section{Research Article}

Keywords: anticoagulants, sepsis, multicenter, DIC, patients

Posted Date: September 9th, 2021

DOI: https://doi.org/10.21203/rs.3.rs-871252/v1 
License: (c) (i) This work is licensed under a Creative Commons Attribution 4.0 International License. Read Full License 


\section{Abstract}

Disseminated intravascular coagulation (DIC) is one of the major organ dysfunctions associated with sepsis. This retrospective secondary analysis comprised data from a prospective multicenter study to investigate the age-related differences in the survival benefit of anticoagulant therapy in sepsis according to the DIC diagnostic criteria. Adult patients with severe sepsis based on the Sepsis-2 criteria were enrolled and divided into the following groups: (1) anticoagulant group (patients who received anticoagulant therapy) and (2) non-anticoagulant group (patients who did not receive anticoagulant therapy). Patients in the former group were administered antithrombin, recombinant human thrombomodulin, and their combination. The multivariate Cox proportional hazard regression model showed that the risk increases were suppressed in the high-DIC-score patients aged 60-70 years receiving anticoagulant therapy. No favorable association of anti-coagulant therapy with hospital mortality was observed in patients aged 50 years and 80 years. Furthermore, anticoagulant therapy in the lower-DIC-score range increased the risk in patients aged 50-60 years. In conclusion, anticoagulant therapy was associated with decreased hospital mortality according to a higher DIC score in septic patients aged 60-70 years. Anticoagulant therapy, however, was not associated with a better outcome in relatively younger and older patients with sepsis.

\section{Introduction}

Disseminated intravascular coagulation (DIC) is characterized by systemic thrombin generation, not restricted to the site of insult, and is followed by microvascular fibrin thrombosis. Since the 1990s, DIC has been known as one of the major organ failures associated with sepsis ${ }^{1,2}$. Moreover, DIC gives rise to multiple organ dysfunction and affects patient outcomes ${ }^{3}$. Large-scale randomized clinical trials (RCTs) have been conducted to verify the effects of anticoagulant agents, including the HETRASE study for unfractionated heparin ${ }^{4}$; the KyberSept trial for antithrombin ${ }^{5}$; the PROWESS, ADDRESS, and PROWESSSHOCK trials for activated protein $\mathrm{C}^{6-8}$; the OPTIMIST and CAPTIVATE trials for tissue factor pathway inhibitor ${ }^{9,10}$; and the SCARLET trial for recombinant human thrombomodulin ${ }^{11}$. However, these abovementioned studies failed to determine the efficacy of anticoagulant therapy against sepsis, which may be attributed to the fact that most of these RCTs targeted heterogeneous patients, such as those with "sepsis" or "severe sepsis"12. Recently, it has become widely apparent that patients with three factors, namely, "sepsis," "DIC," and "high disease severity," may constitute an optimal target for anticoagulant therapies ${ }^{13}$. We previously demonstrated that anticoagulant therapy is associated with better outcomes according to the deterioration of both DIC and disease severity ${ }^{14}$, which were evaluated by the International Society of Thrombosis and Haemostasis (ISTH) overt DIC scoring system ${ }^{1}$ and the Acute Physiology and Chronic Health Evaluation (APACHE) II score, respectively ${ }^{15}$.

The ISTH overt DIC diagnostic criteria have sufficient accuracy for diagnosing DIC. However, it has been pointed out that by the time overt DIC can be identified by the ISTH criteria, the patient may already be in an irreversible and decompensated stage, which may be too late, from a therapeutic perspective, for 
initiating effective interventions ${ }^{3}$. The Japanese Association for Acute Medicine (JAAM) DIC diagnostic criteria, which were established to overcome the limitations of the ISTH overt DIC criteria, have good diagnostic properties and predictive accuracy for 28-day and hospital mortality in patients with severe sepsis $^{16}$. In addition, the JAAM DIC diagnostic criteria can detect twice as many cases in less time than that needed by the ISTH overt DIC criteria ${ }^{17}$. The APACHE II scoring system, which is a classification system for measuring disease severity, comprises three components: age, chronic disease score, and the Acute Physiology Score (APS). APACHE II score has been widely used to predict outcomes, including sepsis, for critically ill patients ${ }^{18}$. However, the APS is defined as a sum of the worst values of 12 physiological indicators within $24 \mathrm{~h}$ after intensive care unit (ICU) admission; thus, considering its simplicity and promptness, the APACHE II score may not be useful for decision-making at the time of treatment initiation. Thus, based on previous evidence that DIC is simultaneously associated with increasing disease severity, we focused on age, which is one of the components of the APACHE II score. Aging is accompanied by an increase in the prothrombotic state ${ }^{19}$, suggesting that age may be a key factor in the pathophysiology of sepsis-associated DIC, affecting the efficacy of anticoagulant therapy against sepsis.

Therefore, this study was conducted, using the nationwide sepsis registry data set, with the aim of examining the age-related differences in the survival benefit conferred by anticoagulant therapy in sepsis, in accordance with the JAAM DIC diagnostic criteria.

\section{Results}

Study Population. In total, 1,184 consecutive patients fulfilling the inclusion criteria were registered during the study period in the FORECAST Sepsis cohort. Six registered patients who had missing values exceeding the threshold $(>170)$ were detected by a one-sample robust regression with an $\mathrm{M}$ estimator. Moreover, 38 patients were excluded due to missing information on the administration of antithrombin and recombinant human thrombomodulin. Subsequently, the data from a final cohort of 1,140 patients were analyzed in the present study. The anticoagulant group comprised 331 patients (antithrombin, 89 patients; recombinant human thrombomodulin, 100; and their combination, 142), and the nonanticoagulant group comprised 809 patients (Fig. 1).

Table 1 shows the baseline clinical characteristics and therapeutic interventions administered to the patients with or without anticoagulant treatments. Patient characteristics, such as age and sex, were similar in the two groups. The CCI, APACHE II, SOFA, and DIC scores were significantly higher in the anticoagulant group than in the non-anticoagulant group. In addition, the anticoagulant group showed higher rates of septic shock and positive blood culture than the non-anticoagulant group. The commonest sites of infection were the lung in the non-anticoagulant group and abdomen in the anticoagulant group, and these rates differed between the two groups. The rates of concomitant therapeutic interventions were also different between the two groups. 
Table 1

Baseline clinical characteristics of the patients who did or did not receive anticoagulant therapy

Non-anticoagulant group Anticoagulant group $P$

$\mathrm{N}=\mathbf{8 0 9} \quad \mathrm{N}=\mathbf{3 3 1}$

Patient characteristics

$\begin{array}{llll}\text { Age, years } & 73(64-82) & 72(64-81) & 0.609 \\ \text { Sex (female/male) } & 38.7 / 61.3(313 / 496) & 41.7 / 58.3(138 / 193) & 0.347\end{array}$

Preexisting conditions

Charlson Comorbidity Index

$1(0-2)$

$1(0-2)$

0.017

ADL Dependent/Independent

25.1/74.9 (203/606)

$21.2 / 78.8(70 / 260)$

0.164

Malignant disease, no/yes

$87.4 / 12.6(707 / 102)$

$(276 / 55)$

0.075

Severe liver disease, no/yes

98.1/1.9 (794/15)

97.0/3.0 (321/10)

0.222

Prescribed anticoagulants, no/yes

90.6/9.4 (733/76)

91.2/8.8 (302/29)

0.737

Illness severity

\begin{tabular}{llll} 
APACHE II score & $21(16-28)$ & $27(20-33)$ & $<0.001$ \\
SOFA score & $8(5-11)$ & $10(7-13)$ & $<0.001$ \\
SIRS score & $3(2-4)$ & $3(2-4)$ & 0.176 \\
\hline ISTH DIC score & $2(1-4)$ & $4(3-5)$ & $<0.001$ \\
\hline JAAM DIC score & $3(2-5)$ & $5(4-6)$ & $<0.001$ \\
Septic shock, no/yes & $44.5 / 55.5(360 / 449)$ & $20.5 / 79.5(68 / 263)$ & $<0.001$ \\
Blood culture, negative/positive & $43.4 / 56.6(349 / 455)$ & $35.3 / 64.7(117 / 214)$ & 0.012 \\
\hline Primary site of infection & & & $<0.001$ \\
Abdomen & $21.6(175)$ & $35.6(118)$ & \\
Lung & $35.6(288)$ & $20.5(68)$ & \\
Urinary tract & $18.8(152)$ & $19.6(65)$ &
\end{tabular}

Data are reported as proportion (count) for categorical variables and median (interquartile range) for continuous variables.

ADL, activities of daily living; APACHE, Acute Physiology and Chronic Health Evaluation; CNS, central nerve system; CRRT, continuous renal replacement therapy; DIC, disseminated intravascular coagulation; IVIg, intravenous immunoglobulin; ISTH, International Society on Thrombosis and Haemostasis; JAAM, Japanese Association for Acute Medicine; PMX-DHP, polymyxin B direct hemoperfusion; SIRS, systemic inflammatory response syndrome; SOFA, Sequential Organ Failure Assessment. 


\begin{tabular}{|c|c|c|c|}
\hline & $\begin{array}{l}\text { Non-anticoagulant group } \\
\mathrm{N}=809\end{array}$ & $\begin{array}{l}\text { Anticoagulant group } \\
\mathrm{N}=331\end{array}$ & $P$ \\
\hline Skin/soft tissue & $9.6(78)$ & $10.3(34)$ & \\
\hline Blood stream & $2.1(17)$ & $1.2(4)$ & \\
\hline Bone/joint & $2.0(16)$ & $1.2(4)$ & \\
\hline CNS & $1.7(14)$ & $2.1(7)$ & \\
\hline Endocardium & $1.4(11)$ & $1.5(5)$ & \\
\hline Implant device & $0.9(7)$ & $0.3(1)$ & \\
\hline Wound & $1.0(8)$ & $0.6(2)$ & \\
\hline Others & $5.3(43)$ & $6.9(23)$ & \\
\hline \multicolumn{4}{|l|}{ Therapeutic interventions } \\
\hline Mechanical ventilation & $52.2 / 47.8(420 / 384)$ & $42.4 / 57.6(140 / 190)$ & 0.003 \\
\hline PMX-DHP & 96.3/3.7 (779/30) & $79.5 / 20.5(263 / 68)$ & $<0.001$ \\
\hline IVlg & $91.1 / 8.9(733 / 72)$ & $54.3 / 45.7(178 / 150)$ & $<0.001$ \\
\hline Protease inhibitor & $95.0 / 5.0(768 / 40)$ & $86.3 / 13.7(283 / 45)$ & $<0.001$ \\
\hline CRRT & $82.5 / 17.5(664 / 141)$ & $51.7 / 48.3(171 / 160)$ & $<0.001$ \\
\hline Corticosteroids & $77.4 / 22.6(625 / 182)$ & $51.2 / 48.8(169 / 161)$ & $<0.001$ \\
\hline Noradrenaline & $41.1 / 58.9(332 / 475)$ & 19.0/81.0 (63/268) & $<0.001$ \\
\hline Enteral nutrition & $55.8 / 44.2(450 / 357)$ & 49.8/50.2 (164/165) & 0.070 \\
\hline \multicolumn{4}{|c|}{$\begin{array}{l}\text { Data are reported as proportion (count) for categorical variables and median (interquartile range) for } \\
\text { continuous variables. }\end{array}$} \\
\hline \multicolumn{4}{|c|}{$\begin{array}{l}\text { ADL, activities of daily living; APACHE, Acute Physiology and Chronic Health Evaluation; CNS, central } \\
\text { nerve system; CRRT, continuous renal replacement therapy; DIC, disseminated intravascular } \\
\text { coagulation; IVIg, intravenous immunoglobulin; ISTH, International Society on Thrombosis and } \\
\text { Haemostasis; JAAM, Japanese Association for Acute Medicine; PMX-DHP, polymyxin B direct } \\
\text { hemoperfusion; SIRS, systemic inflammatory response syndrome; SOFA, Sequential Organ Failure } \\
\text { Assessment. }\end{array}$} \\
\hline
\end{tabular}

Effect of Anticoagulant Therapy on Hospital Mortality. The overall cumulative survival probability that was obtained in all patients included in this study is shown in Fig. 2. The hospital mortality rate was $30.2 \%(100 / 331)$ in the anticoagulant group and $20.5 \%(166 / 809)$ in the non-anticoagulant group. The multivariable regression model showed that the hazard ratio (anticoagulant/non-anticoagulant) of hospital mortality was 0.910 (upper 0.665 , lower $1.245, P=0.554$ ). 
We performed multivariate Cox proportional hazard regression model analysis, which included a two-way interaction, to identify the effect modification of anticoagulant therapy by the DIC score (Fig. 3A). Neither anticoagulant therapy nor the DIC score showed a significant interaction with hospital mortality $(P=$ 0.773 and $P=0.777$, respectively). The $P$-value for the two-way interaction was 0.687 . The multivariate Cox proportional hazard regression model including the three-way interaction term between the previous two (anticoagulant therapy and DIC score) and age, which may influence the therapeutic effect, showed that the increases in the risk were suppressed in the population with higher DIC scores in patients aged 60 to 70 years in the anticoagulant group. In patients aged 50 years with sepsis, the risk in the nonanticoagulant group tended to increase concomitantly with increases in the DIC score in the low-score range (DIC score $<4$ ), whereas no increase in the risk was observed in the high-score range (DIC score $\geq$ 4), and a favorable association of anti-coagulant therapy with hospital mortality was not found. In patients aged 80 years, the non-anticoagulant group indicated a certain risk regardless of the DIC score, and the anti-coagulant therapy showed no beneficial effect through a decreased risk of hospital mortality. Importantly, anticoagulant therapy in the lower DIC score range increased the risk hazard in patients aged 50 to 60 years. Global $P$-values for age and the three-way interaction were 0.041 and 0.055 , respectively (Fig. 3B).

SOFA score $72 \mathrm{~h}$ after admission according to the DIC score. The multivariate linear regression model was used to elucidate the effect modification of anticoagulant therapy on organ dysfunction by DIC score (Fig. 4). The risk hazard of the SOFA score $72 \mathrm{~h}$ after admission in the non-anticoagulant group increased concomitantly with an increase in DIC score. Anticoagulant therapy showed no favorable association with the SOFA score. In addition, when we considered the population with lower DIC scores, a higher risk of increase in the SOFA score was found in the anticoagulant group (Fig. 4A). We assessed the agerelated effect of anticoagulant therapy on the SOFA score as well as on hospital mortality (Fig. 4B). Anticoagulant therapy did not improve the SOFA score in any age group, and therapy tended to induce a deterioration in SOFA scores in the low-DIC-score range.

\section{Discussion}

We investigated the age-related differences in the survival benefit of anticoagulant therapy in sepsis in accordance with the JAAM DIC diagnostic criteria. The results of this study indicate that anticoagulant therapy is associated with better outcomes consistent with a higher DIC score in patients aged 60 to 70 years. Moreover, anticoagulant therapy in patients with sepsis and low DIC scores may be associated with the deterioration of organ function and could lead to poor outcomes.

Previous studies have demonstrated that patients with sepsis and DIC exhibit an increased prevalence of multiple organ dysfunction and that the mortality rate of such patients is significantly higher than that of non-DIC patients ${ }^{16,20}$, suggesting that the development of DIC in sepsis is an indicator of poor prognosis. A recent nationwide multicenter retrospective cohort study suggested that screening for DIC was associated with a survival benefit in patients with sepsis ${ }^{21}$. These results imply that the evaluation and subsequent intervention for coagulofibrinolytic changes related to sepsis may contribute to improved 
outcomes in patients with sepsis. To date, however, there is no clear evidence that treating sepsis-induced coagulopathy leads to improved outcomes in patients with sepsis.

The important point is that most of the RCTs that evaluated the effect of anticoagulant therapy have targeted patients with "sepsis." Although the KyberSept trial, a mega-RCT, demonstrated that antithrombin treatment did not affect 28-day mortality in adult patients with sepsis and septic shock ${ }^{5}$, its post hoc analysis suggested that antithrombin therapy was associated with a significant reduction in mortality only in patients with DIC but not in non-DIC patients ${ }^{22}$. These results indicate the importance of selecting the target population for anticoagulant therapy against sepsis. Studies showing that the optimal target for anticoagulant therapy is a patient population fulfilling three factors, namely, sepsis, DIC, and high disease severity, have recently been published ${ }^{13,14,23-25}$.

A recent global analysis demonstrated that 1 in 5 deaths around the world is caused by sepsis, leading to 11 million deaths annually worldwide, thus surpassing cancer mortality ${ }^{26}$. Older people constitute the major population of patients with sepsis, and mortality increases at more advanced ages ${ }^{27,28}$. Although it is well known that advancing age is associated with increased coagulation, this propensity for a deteriorating coagulation disorder during sepsis remains unknown ${ }^{27}$. Innate immune cells, such as neutrophils and macrophages, show age-related functional alteration, resulting in a diminished ability to rapidly respond to foreign pathogens ${ }^{29}$. In addition to the innate immune system, an attenuation of the adaptive immune functions in old age has been confirmed ${ }^{30}$. A prolonged inflammatory response in aged patients has been noted, as high levels of inflammatory cytokines have been found ${ }^{31-34}$. The interaction between the effect of anticoagulant therapy and the DIC score in patients aged 80 years that was found in our study may support these previous studies. In other words, the onset of sepsis itself may determine the outcome regardless of the severity of DIC in the elderly. In contrast, patients aged 50 years showed a different interaction between anticoagulant therapy and the DIC score. Relatively young patients with sepsis are expected to respond well to the treatment of underlying infection, followed by the modulation of dysregulated inflammatory and coagulofibrinolytic reactions. Therefore, it is presumed that the development of DIC in relatively young patients with sepsis may have minimal effect on the outcome, although the detailed underlying mechanisms remain unclear.

The present study failed to show a beneficial effect of anticoagulant therapy on the SOFA score of the population in any age group. However, this does not mean that organ dysfunction cannot be improved by anticoagulant therapy, because the SOFA scores were evaluated relatively early (72 $\mathrm{h}$ after admission). As anticoagulant therapy improved hospital mortality in certain ages, it may improve SOFA scores later than $72 \mathrm{~h}$ after admission. Our results should be considered when setting up the study designs of future RCTs to validate the effects of anticoagulant therapy against sepsis.

The main pathophysiology of DIC is uncontrolled thrombin generation, leading to ischemic organ dysfunction due to microvascular thrombosis, which is detrimental in the context of pathology. In contrast, the concept that microvascular thrombosis produced by innate immunity is a physiological 
process to maintain body homeostasis has come to be known as immunothrombosis ${ }^{3,35}$. From these perspectives, individuals who could be at an advantage with regard to immunothrombosis, namely, nonDIC patients, should not be treated with anticoagulant therapy. The harmful effects of anticoagulant therapy against non-DIC patients were confirmed in the present study (Figs. 3 and 4).

The current study has several limitations. First, although the present data set was prospectively collected, causal relationships could not be defined because of the study's retrospective design. Second, this study did not assess the dosage and duration of anticoagulant agents. Additionally, this study was unable to evaluate the effects of individual anticoagulant agents or combination therapy because we defined anticoagulant therapy as the administration of antithrombin, recombinant human thrombomodulin, or their combination. Third, the present study data set did not include information on adverse effects, including serious bleeding complications associated with anticoagulant therapy, which can cause unfavorable outcomes. Fourth, data elements required to control potential confounders might have resulted in biased effect estimates. Finally, the study being conducted in a single country may limit the generalizability of the obtained results.

In conclusion, anticoagulant therapy, namely, the administration of antithrombin, recombinant human thrombomodulin, or their combination, showed a beneficial effect on hospital mortality according to a higher JAAM DIC score in patients aged 60 to 70 years with sepsis. Anticoagulant therapy, however, was not associated with a better outcome in relatively young (50 years) and older (80 years) patients. In addition, anticoagulant therapy in patients with low DIC scores was associated with the deterioration of organ function and poor outcomes, which may be caused by the destruction of the physiological hypercoagulative state and immunothrombosis. We suggest setting the inclusion criteria of future RCTs examining the effects of anticoagulant therapy against sepsis based on the results obtained from the present study.

\section{Methods}

Study design, setting, and ethical approval. This study was a retrospective secondary analysis of a sepsis cohort of the prospective, multicenter JAAM Focused Outcomes Research in Emergency Care in Acute Respiratory Distress Syndrome, Sepsis and Trauma (FORECAST) study ${ }^{36}$. The JAAM FORECAST Sepsis study was conducted from January 2016 to March 2017 and used consecutive samples from 59 ICUs in Japan. The study was registered in the University Hospital Medical Information Network Clinical Trial Registry (UMIN-CTR ID: UMIN000019588). This study was approved by the JAAM and Ethics Committee of each hospital (JAAM, 2014-01; Hokkaido University Faculty of Medicine, Head institute of the FORECAST group, 014-0307) after written informed consent was obtained from each patient or their next of kin and was performed in accordance with the tenets underlying the Declaration of Helsinki.

Participants. The JAAM FORECAST Sepsis study enrolled adult patients (aged $>16$ years) who had been admitted to the ICU with severe sepsis and septic shock according to the Sepsis-2 criteria published in $2003^{37}$, based on the following inclusion criteria: (i) patients suspected to have or who were diagnosed 
with new-onset infection based on the history of the present illness; (ii) patients who met $\geq 2$ systemic inflammatory response syndrome (SIRS) criteria; and (iii) patients who had at least one organ dysfunction, defined by the following criteria: systolic blood pressure $<90 \mathrm{mmHg}$, mean arterial pressure $<65 \mathrm{mmHg}$, or decreased blood pressure $>40 \mathrm{mmHg}$ at baseline; serum creatinine $>2.0 \mathrm{mg} / \mathrm{dL}$ or urine output $<0.5 \mathrm{~mL} / \mathrm{kg} / \mathrm{h}$; total bilirubin $>2.0 \mathrm{mg} / \mathrm{dL}$; platelet counts $<100 \times 10^{9} / \mathrm{L}$; lactate $>2 \mathrm{mmol} / \mathrm{L}$; prothrombin time International Normalized Ratio (INR) $>1.5$; and arterial hypoxemia $\left(\mathrm{PaO}_{2} / \mathrm{FiO}_{2}\right)<200$ with pneumonia or $\mathrm{PaO}_{2} / \mathrm{FiO}_{2}<250$ without pneumonia. Patients on end-of-life care or those who were resuscitated following cardiac arrest at the time of the diagnosis of severe sepsis were excluded. Patients with substantial missing data were also excluded from the analysis. The size of the study population was dependent on the study period. All patients were followed up until discharge.

Participants were divided into two cohorts: the anticoagulant group and non-anticoagulant group. The anticoagulant group comprised patients who received anticoagulant therapy, defined as the administration of antithrombin, recombinant human thrombomodulin, or their combination, in accordance with the Japanese Clinical Practice Guidelines for Management of Sepsis and Septic Shock $2020^{38}$. The non-anticoagulant group comprised patients who received neither antithrombin nor recombinant human thrombomodulin. There was no pre-determined definitive protocol for the indication of anticoagulant therapy, which was initiated at the discretion of the participating physicians based on the institutional treatment policies at each hospital. The standard dosage and administration of antithrombin and recombinant human thrombomodulin for sepsis-induced DIC in Japan is $1,500 \mathrm{U} /$ day or $30 \mathrm{U} / \mathrm{kg} /$ day, $3-5$ days and $380 \mathrm{U} / \mathrm{kg}, 6$ days, respectively.

\section{Definitions}

We defined SIRS, sepsis, severe sepsis, and septic shock based on the American College of Chest Physicians/Society of Critical Care Medicine consensus conference (Sepsis-1) published in $1992^{39}$ and the revised version (Sepsis-2) published in $2003^{37}$. Disease severity was assessed according to the APACHE II score ${ }^{15}$. Organ dysfunction was evaluated according to the Sequential Organ Failure Assessment (SOFA) score ${ }^{40}$. The Charlson Comorbidity Index (CCI) was adopted for the assessment of baseline comorbidities ${ }^{41}$. DIC was diagnosed based on the JAAM DIC scoring system using prothrombin time INR as a substitute for the prothrombin time ratio (Supplementary Table S1) ${ }^{42}$.

Data Collection. An electronic data capture system was developed for use in this FORECAST study, and data compiled by the FORECAST investigators were obtained from the FORECAST database. Patient information included the baseline characteristics, various comorbidities, activities of daily living, suspected sites of infection, organ dysfunction, sepsis-related severity scores, and therapeutic interventions. Moreover, we obtained data on compliance with established sepsis care protocols, such as the measurement of serum lactate within $3 \mathrm{~h}$ of hospital arrival. The primary outcome was hospital allcause mortality. The SOFA score $72 \mathrm{~h}$ after admission was recorded as the secondary outcome. 
Statistical Analyses. All baseline clinical and demographical characteristics are expressed as median and interquartile range for continuous variables, whereas numbers and percentages are used for categorical variables. For comparisons of these characteristics between the patients who were treated with and without anticoagulants, the Mann-Whitney $U$ test and chi-square test were used for continuous and categorical variables, respectively.

To examine whether the effect of the anticoagulants on hospital mortality was modified by the DIC score, we used a multivariable Cox proportional hazard regression model that included a cross-product term between the anticoagulants' variable and DIC score in addition to the main effect terms as the explanatory variables. The non-linear effect of the DIC score was assessed using a restricted-cubic-spline method with $k n o t, k=3$. Furthermore, we assessed the patient's age as the effect modifier using a similar regression model that included three- and two-way cross-product terms between the variables that indicated the effect of anticoagulants, including the DIC score and age. Moreover, similar analyses were performed for $72 \mathrm{~h}$ SOFA score as the explanatory variable using multivariable non-linear regression models, which were adjusted for all covariates that are described in Table 1. We confirmed that these regression models were not overfitted using bootstrap validation (index-corrected calibration slopes were approximately 0.8 ). The missing values were imputed using a multiple imputation method.

All hypothesis testing was performed with a two-sided, $5 \%$ significance level using $\mathrm{R}$ software (https://cran.r-project.org/).

\section{Declarations}

\section{Data availability statement}

The data that support the findings of this study are available from the authors upon reasonable request.

\section{Acknowledgements}

The JAAM FORECAST Study Group thanks Shuta Fukuda for his special assistance in completing the study. We would like to thank Editage (www.editage.com) for English language editing.

We wish to express our gratitude to all JAAM FORECAST Study investigators: Osaka City University Hospital (Yasumitsu Mizobata); National Hospital Organization Sendai Medical Center (Yasuo Yamada); Saitama Medical University Saitama Medical Center (Satoru Sugiyama); Fukui Prefectural Hospital (Hiroshi Ishida); Sapporo Medical University (Eichi Narimatsu); Fukuyama City Hospital (Koji Miyasho); Hiratsuka City Hospital (Toshio Kanai); Saiseikai Utsunomiya Hospital (Satoru Miyatake); Japanese Red Cross Society Kyoto Daini Hospital (Ryouji liduka); Shinsyu University School of Medicine (Hiroshi Imamura); Rinku General Medical Center (Yasuaki Mizushima); Subaru Health Insurance Society Ota Memorial Hospital (Yoshitake Sato); Saitama Medical University International Medical Center (Manabu Nemoto); Aomori Prefectural Central Hospital (Hiroyuki Hanada); National Hospital Organization Hokkaido Medical Center (Yasuo Shichinohe); Saga-ken Medical Centre Koseikan (Kenji Hirahara); 
Hachinohe City Hospital (Akihide Kon); Juntendo University Nerima Hospital (Manabu Sugita); Kawaguchi Municipal Medical Center (Yasutaka Naoe); Kakogawa West City Hospital (Manabu Kirita); Osaka National Hospital (Daikai Sadamitsu); Kouseiren Takaoka Hospital (Masahiro Yoshida); Hokkaido University Faculty of Medicine (Takeshi Wada); Kameda Medical Center (Atsushi Shiraishi); Sapporo Higashi Tokushukai Hospital (Satoshi Gando); Osaka Medical College (Kazuma Yamakawa); Keio University School of Medicine (Seitaro Fujishima, Junichi Sasaki); National Defence Medical College (Daizoh Saitoh); Tohoku University Graduate School of Medicine (Shigeki Kushimoto); Osaka University Graduate School of Medicine (Hiroshi Ogura); Tsukuba Memorial Hospital (Toshikazu Abe); University of Occupational and Environmental Health (Toshihiko Mayumi); Kobe University Graduate School of Medicine (Joji Kotani); Aichi Medical University Hospital (Naoshi Takeyama); Yamaguchi University Hospital (Ryosuke Tsuruta); Kawasaki Municipal Hospital (Kiyotsugu Takuma); Kurume University (Norio Yamashita); Aizu Chuo Hospital (Shin-ichiro Shiraishi); Teikyo University School of Medicine (Hiroto Ikeda); Kawasaki Medical School (Yasukazu Shiino); Kyorin University School of Medicine (Takehiko Tarui); Chiba University Graduate School of Medicine (Takaaki Nakada); St. Luke's International Hospital (Toru Hifumi); Kitakyushu City Yahata Hospital (Kohji Okamoto); Saga University Hospital (Yuichiro Sakamoto); Center Hospital of the National Center for Global Health and Medicine (Akiyoshi Hagiwara); Nippon Medical School (Tomohiko Masuno); Community Healthcare Organization, Chukyo Hospital (Masashi Ueyama); Osaka General Medical Center (Satoshi Fujimi, Yutaka Umemura); and Tokyo Medical and Dental University (Yasuhiro Otomo).

\section{Authors' Contributions}

T.W. conceived and designed this study; contributed to the analysis and interpretation of the data; and was responsible for drafting, editing, and submission of the manuscript. K.Y. contributed to the acquisition of data, interpreted the data, and revised the manuscript for important intellectual content. D.K. played a significant role in data analysis and helped to draft the manuscript. A.S. contributed to data acquisition, conducted data cleaning, interpreted the data, and revised the manuscript for important intellectual content. S.G. had a significant influence on data interpretation and the critical appraisal of the manuscript. T.A., H.O., D.S., S.K., S.F., T.M., T.H., Y.S., T.N., T.T., Y.O., K.O., Y.U., J.K., Y.S., J.S., S.S., K.T., R.T., A.H., T.M., N.T., N.Y., H.I., M.U., and S.F. contributed to the design of the study, organization of data collection, drafting of database charts, and scientific discussion of all processes of the study as well as reviewed the manuscript and registered the patients. All authors approved the final version of the manuscript.

\section{Competing Interests}

Satoshi Gando reported receiving personal fees from Grifols outside the submitted work. Atsushi Shiraishi reported receiving personal fees from CSL Behring outside the submitted work. The other authors declare no competing interests.

\section{References}


1. Taylor, F. B. Jr. et al. Scientific Subcommittee on Disseminated Intravascular Coagulation (DIC) of the International Society on Thrombosis and Haemostasis (ISTH). Towards definition, clinical and laboratory criteria, and a scoring system for disseminated intravascular coagulation. Thromb. Haemost, 86, 1327-1330 (2001).

2. Bone, R. C. The pathogenesis of sepsis. Ann. Intern. Med, 115, 457-469 (1991).

3. Gando, S., Levi, M. \& Toh, C. H. Disseminated intravascular coagulation. Nat. Rev. Dis. Primers, 2, 16037 (2016).

4. Jaimes, F. et al. Unfractioned heparin for treatment of sepsis: a randomized clinical trial (The HETRASE Study). Crit. Care Med, 37, 1185-1196 (2009).

5. Warren, B. L. et al. KyberSept Trial Study Group. Caring for the critically ill patient. High-dose antithrombin III in severe sepsis: a randomized controlled trial. JAMA, 286, 1869-1878 (2001).

6. Ranieri, V. M. et al. Drotrecogin alfa (activated) in adults with septic shock. N. Engl. J. Med, 366, 2055-2064 (2012).

7. Bernard, G. R. et al. Recombinant human protein C Worldwide Evaluation in Severe Sepsis (PROWESS) study group. Efficacy and safety of recombinant human activated protein $\mathrm{C}$ for severe sepsis. N. Engl. J. Med, 344, 699-709 (2001).

8. Abraham, E. et al. Administration of Drotrecogin Alfa (Activated) in Early Stage Severe Sepsis (ADDRESS) Study Group. Drotrecogin alfa (activated) for adults with severe sepsis and a low risk of death. N. Engl. J. Med, 353, 1332-1341 (2005).

9. Abraham, E. et al. Efficacy and safety of tifacogin (recombinant tissue factor pathway inhibitor) in severe sepsis: a randomized controlled trial. JAMA, 290, 238-247 (2003).

10. Wunderink, R. G. et al. CAPTIVATE Trial Group. Recombinant tissue factor pathway inhibitor in severe community-acquired pneumonia: a randomized trial. Am. J. Respir. Crit. Care Med, 183, 1561-1568 (2011).

11. Vincent, J. L. et al. Effect of a recombinant human soluble thrombomodulin on mortality in patients with sepsis-associated coagulopathy: the SCARLET randomized clinical trial. JAMA, 321, 19932002 (2019).

12. Grimaldi, D. \& Vincent, J. L. Clinical trial research in focus: rethinking trials in sepsis. Lancet Respir. Med, 5, 610-611 (2017).

13. Umemura, Y. \& Yamakawa, K. Optimal patient selection for anticoagulant therapy in sepsis: an evidence-based proposal from Japan. J. Thromb. Haemost, 16, 462-464 (2018).

14. Yamakawa, K. et al. Identifying sepsis populations benefitting from anticoagulant therapy: a prospective cohort study incorporating a restricted cubic spline regression model. Thromb. Haemost, 119, 1740-1751 (2019).

15. Knaus, W. A., Draper, E. A., Wagner, D. P. \& Zimmerman, J. E. APACHE II: a severity of disease classification system. Crit. Care Med, 13, 818-829 (1985). 
16. Gando, S. et al. A multicenter, prospective validation study of the Japanese Association for Acute Medicine disseminated intravascular coagulation scoring system in patients with severe sepsis. Crit. Care, 17, R111 (2013).

17. Iba, T. et al. Japanese Surviving Sepsis Campaign Guideline Working Group for disseminated intravascular coagulation. Diagnosis of sepsis-induced disseminated intravascular coagulation and coagulopathy. Acute Med. Surg, 6, 223-232 (2019).

18. Delibegovic, S., Markovic, D. \& Hodzic, S. APACHE II scoring system is superior in the prediction of the outcome in critically ill patients with perforative peritonitis. Med. Arh, 65, 82-85 (2011).

19. Wilkerson, W. R. \& Sane, D. C. Aging and thrombosis. Semin. Thromb. Hemost, 28, 555-568 (2002).

20. Gando, S. et al. Role of disseminated intravascular coagulation in severe sepsis. Thromb. Res, 178, 182-188 (2019).

21. Umemura, Y., Yamakawa, K., Hayakawa, M., Hamasaki, T. \& Fujimi, S. Japan Septic Disseminated Intravascular Coagulation (J-Septic DIC) study group. Screening itself for disseminated intravascular coagulation may reduce mortality in sepsis: a nationwide multicenter registry in Japan. Thromb. Res, 161, 60-66 (2018).

22. Kienast, J. et al. KyberSept investigators. Treatment effects of highdose antithrombin without concomitant heparin in patients with severe sepsis with or without disseminated intravascular coagulation. J. Thromb. Haemost, 4, 90-97 (2006).

23. Umemura, Y., Yamakawa, K., Ogura, H., Yuhara, H. \& Fujimi, S. Efficacy and safety of anticoagulant therapy in three specific populations with sepsis: a meta-analysis of randomized controlled trials. $J$. Thromb. Haemost, 14, 518-530 (2016).

24. Yamakawa, K. et al. Benefit profile of anticoagulant therapy in sepsis: a nationwide multicentre registry in Japan. Crit. Care, 20, 229 (2016).

25. Yoshimura, J. et al. Benefit profile of recombinant human soluble thrombomodulin in sepsis-induced disseminated intravascular coagulation: a multicenter propensity score analysis. Crit. Care, 19, 78 (2015).

26. Rudd, K. E. et al. Global, regional, and national sepsis incidence and mortality, 1990-2017: analysis for the Global Burden of Disease Study. Lancet, 395, 200-211 (2020).

27. Starr, M. E. \& Saito, H. Sepsis in old age: review of human and animal studies. Aging Dis, 5, 126-136 (2014).

28. Angus, D. C. et al. Epidemiology of severe sepsis in the United States: analysis of incidence, outcome, and associated costs of care. Crit.Care Med, 29, 1303-1310 (2001).

29. Gomez, C. R., Nomellini, V., Faunce, D. E. \& Kovacs, E. J. Innate immunity and aging. Exp. Gerontol, 43, 718-728 (2008).

30. Weksler, M. E., Goodhardt, M. \& Szabo, P. The effect of age on B cell development and humoral immunity. Springer Semin. Immunopathol, 24, 35-52 (2002). 
31. Kale, S. et al. GenIMS Investigators. The effects of age on inflammatory and coagulation-fibrinolysis response in patients hospitalized for pneumonia. PLoS One, 5, e13852 (2010).

32. Naffaa, M. et al. Interleukin- 6 at discharge predicts all-cause mortality in patients with sepsis. Am. J. Emerg. Med, 31, 1361-1364 (2013).

33. Marik, P. E., Zaloga, G. P. \& NORASEPT II Study Investigators. The effect of aging on circulating levels of proinflammatory cytokines during septic shock. Norasept II Study Investigators. J. Am. Geriatr. Soc, 49, 5-9 (2001).

34. Bruunsgaard, H., Skinhøj, P., Qvist, J. \& Pedersen, B. K. Elderly humans show prolonged in vivo inflammatory activity during pneumococcal infections. J. Infect. Dis, 180, 551-554 (1999).

35. Engelmann, B. \& Massberg, S. Thrombosis as an intravascular effector of innate immunity. Nat. Rev. Immunol, 13, 34-45 (2013).

36. Abe, T. et al. Characteristics, management, and in-hospital mortality among patients with severe sepsis in intensive care units in Japan: the FORECAST study. Crit. Care, 22, 322 (2018).

37. Levy, M. M. et al.. 2001 SCCM/ESICM/ACCP/ATS/SIS International Sepsis Definitions Conference. Intensive Care Med. 29, 530-538 (2003).

38. Egi, M. et al. The Japanese Clinical Practice Guidelines for Management of Sepsis and Septic Shock 2020 (J-SSCG 2020). J. Intensive Care, 9, 53 (2021).

39. American College of Chest Physicians/Society of Critical Care Medicine Consensus Conference: definitions for sepsis and organ failure and guidelines for the use of innovative therapies in sepsis. Crit. Care Med. 20, 864-874 (1992).

40. Vincent, J. L. et al. The SOFA (Sepsis-related Organ Failure Assessment) score to describe organ dysfunction/failure. On behalf of the Working Group on Sepsis-Related Problems of the European Society of Intensive Care Medicine. Intensive Care Med, 22, 707-710 (1996).

41. Charlson, M. E., Pompei, P., Ales, K. L. \& MacKenzie, C. R. A new method of classifying prognostic comorbidity in longitudinal studies: development and validation. J. Chronic Dis, 40, 373-383 (1987).

42. Gando, S. et al. Natural history of disseminated intravascular coagulation diagnosed based on the newly established diagnostic criteria for critically ill patients: results of a multicenter, prospective survey. Crit. Care Med, 36, 145-150 (2008).

\section{Figures}




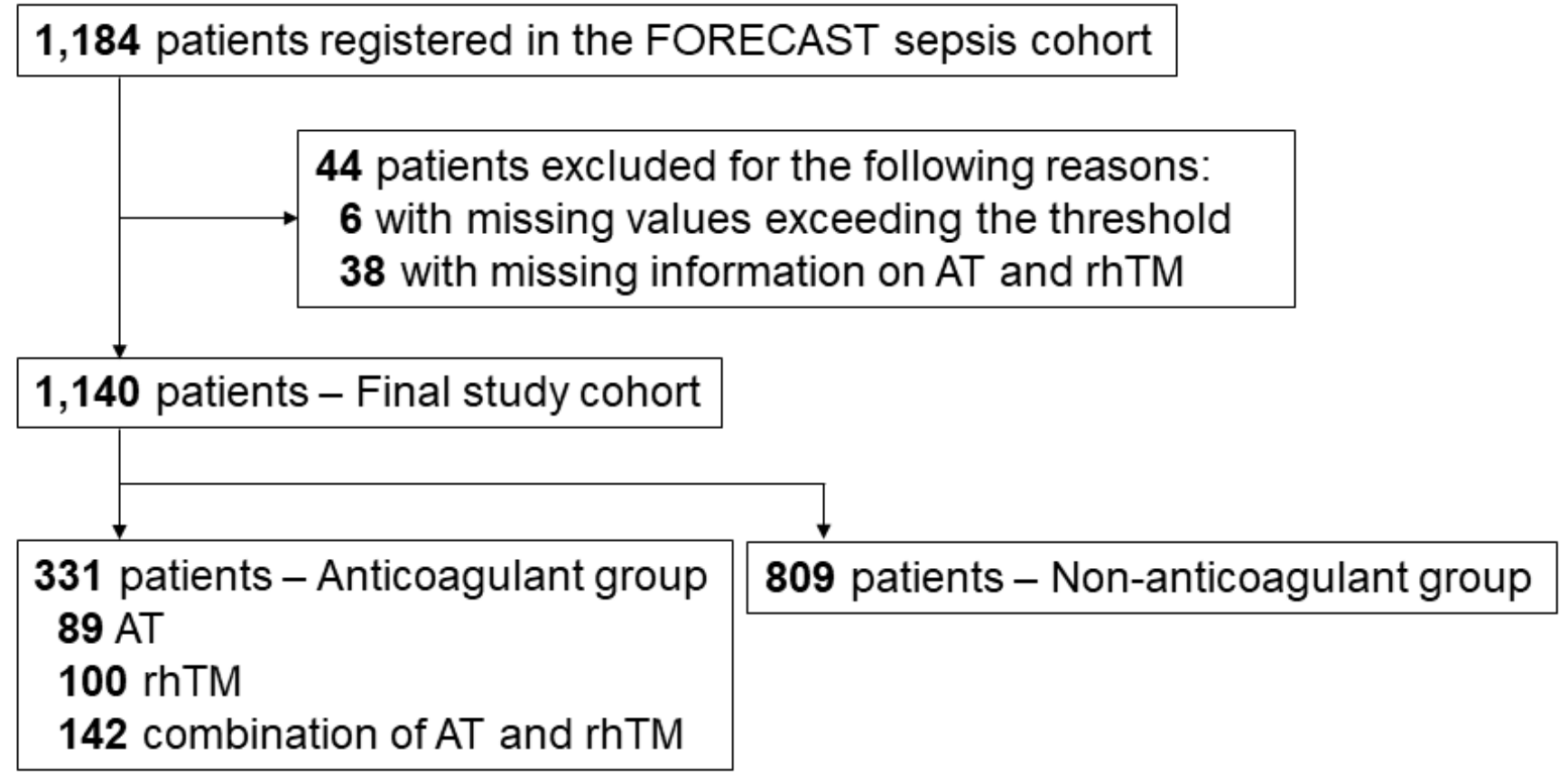

Figure 1

Flow chart of the study population AT, antithrombin; FORECAST, Focused Outcomes Research in Emergency Care in Acute Respiratory Distress Syndrome, Sepsis and Trauma; rhTM, recombinant human thrombomodulin. 


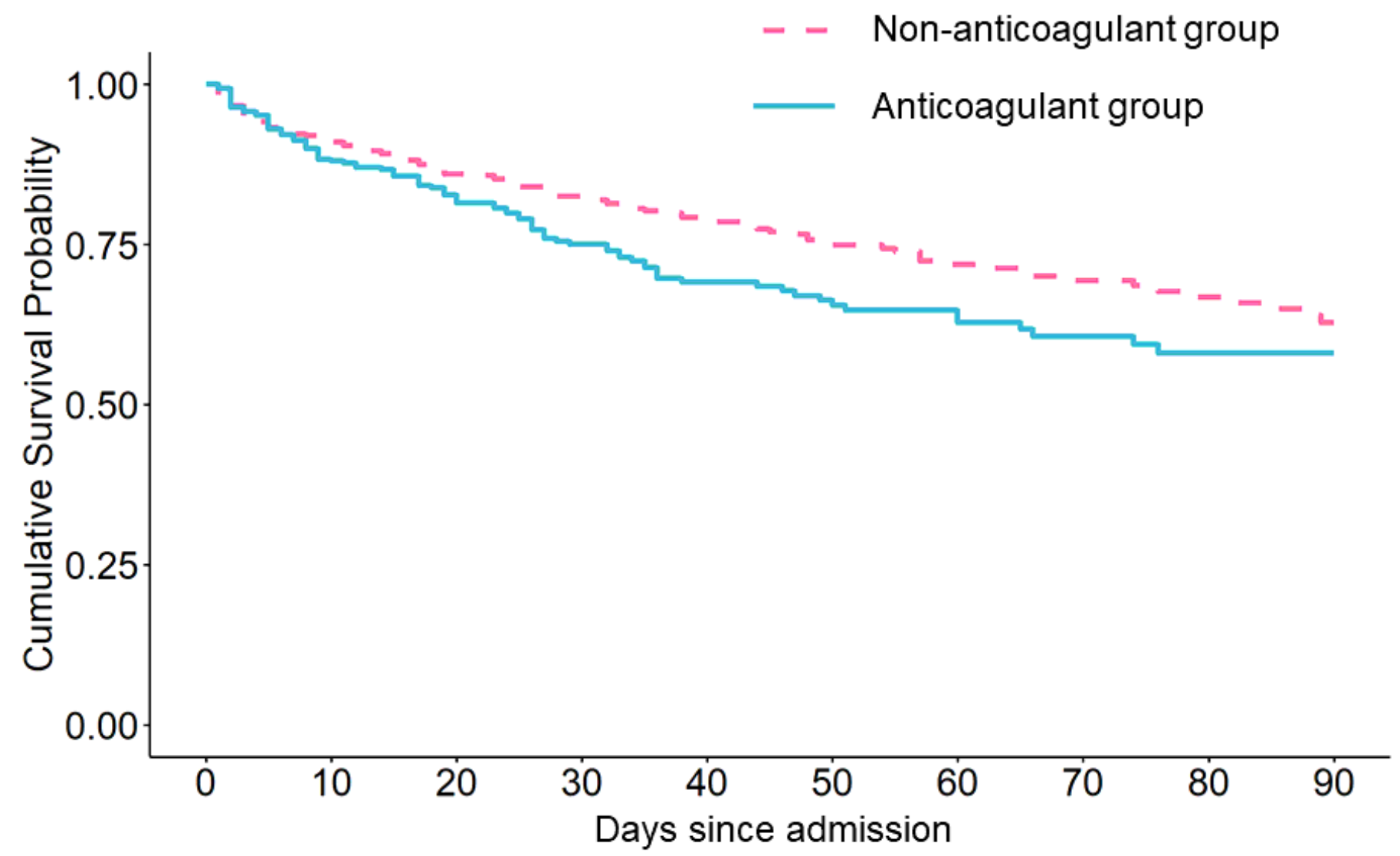

Number at Risk

\begin{tabular}{|c|c|c|c|c|c|c|c|c|}
\hline 809 & 645 & 449 & 312 & 222 & 169 & 129 & 98 & 74 \\
\hline 330 & 276 & 213 & 156 & 111 & 86 & 69 & 52 & 37 \\
\hline
\end{tabular}

Figure 2

The Kaplan-Meier curves during the first 90 days for the cumulative survival of patients with and without anticoagulant therapy The blue line represents the anticoagulant group and the dotted red line represents the non-anticoagulant group. 
$=\quad$ Non-anticoagulant group

(a)

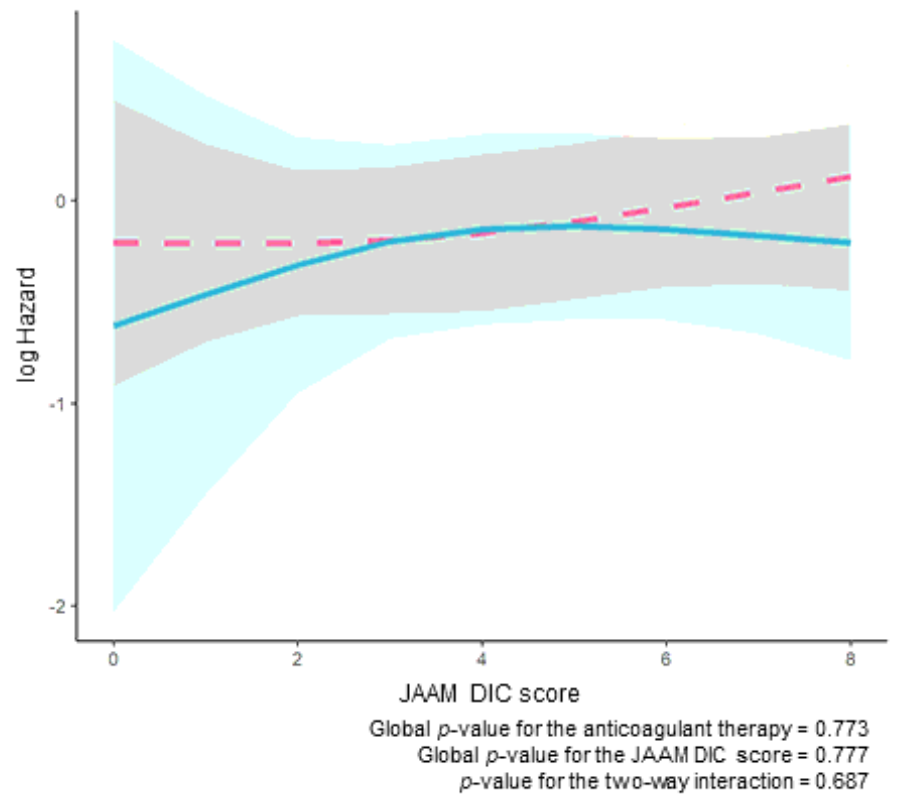

(b)

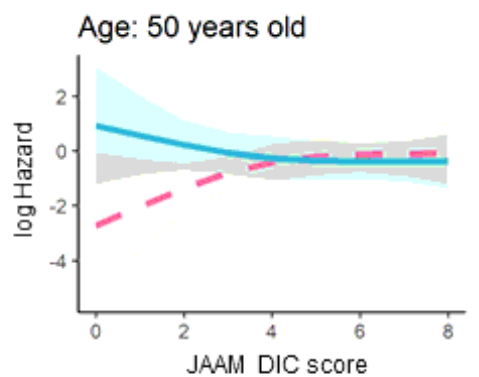

Age: 70 years old
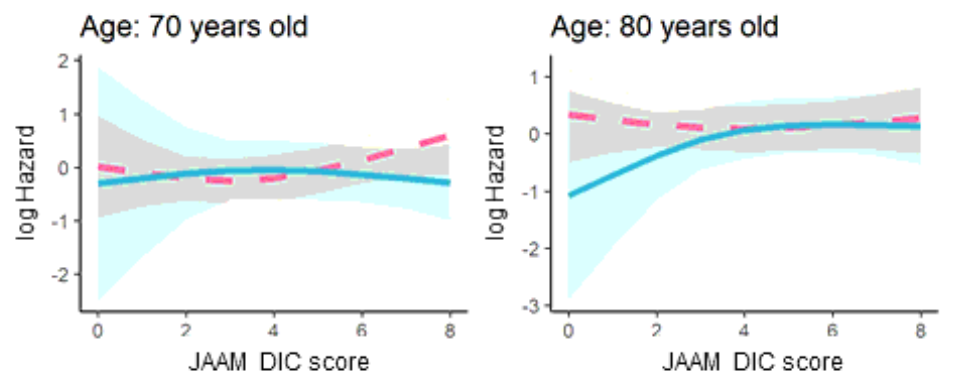

Global p-value for the anticoagulant therapy $=0.18$ Global $p$-value for the JAAM DIC score $=0.415$ Global $p$-value for age $=0.041$ p-value for the three-way interaction $=0.055$

\section{Figure 3}

Regression line of hospital mortality of each treatment group estimated by the Cox proportional hazard regression model. (a) Two-way interaction term between the treatment and JAAM DIC score. (b) Threeway interaction term among anticoagulant therapy, the JAAM DIC score, and age. The lines indicate estimated log-transformed relative hazards, and the shaded areas represent $95 \%$ confidence intervals. The solid blue line represents patients in the anticoagulant group, and the dotted red line represents those in the non-anticoagulant group. JAAM, Japanese Association for Acute Medicine; DIC, disseminated intravascular coagulation. 


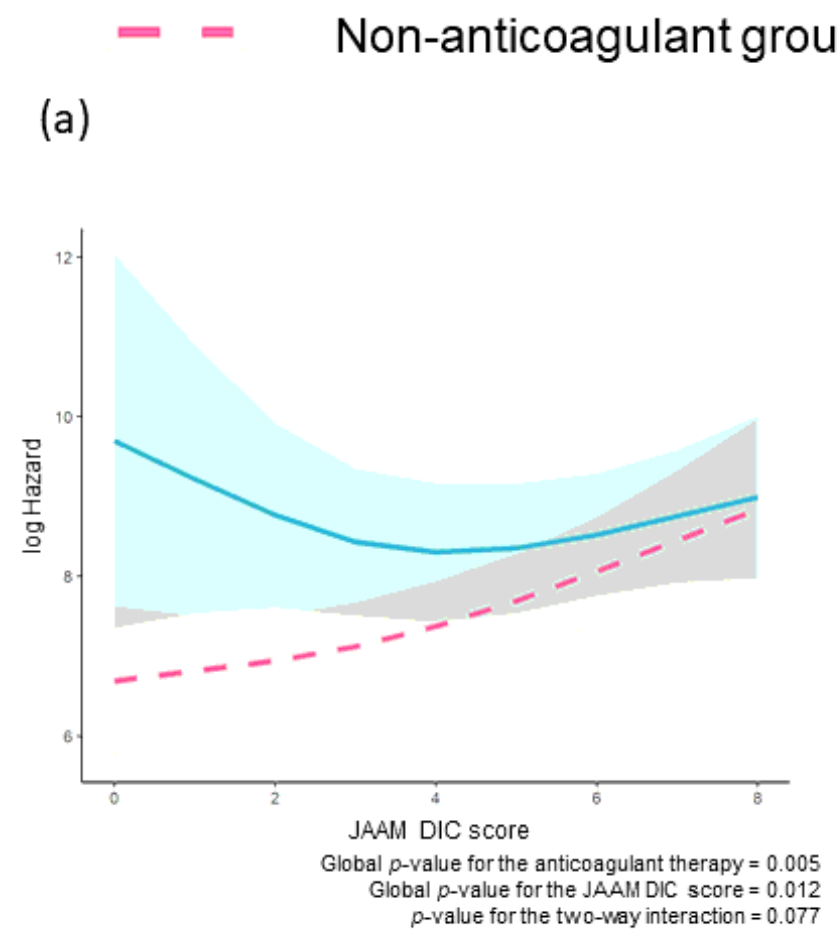

(b)
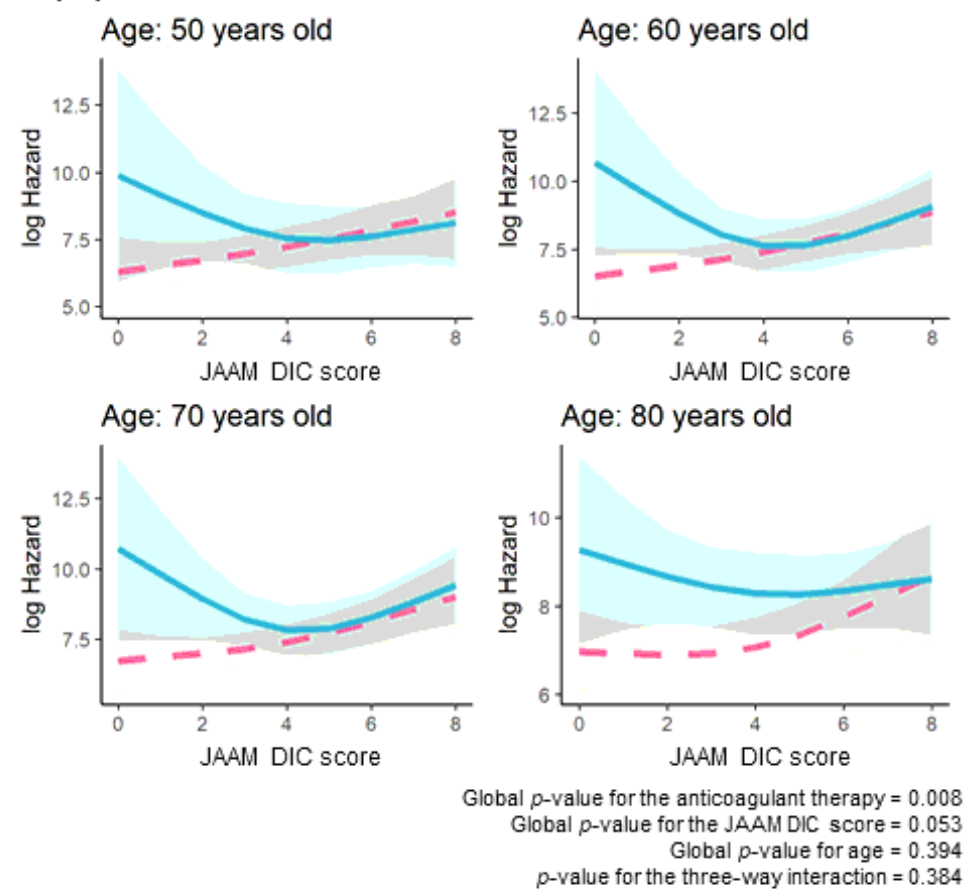

\section{Figure 4}

Regression lines of the SOFA score $72 \mathrm{~h}$ after admission in each treatment group. (a) Two-way interaction term between the treatment and JAAM DIC score. (b) Three-way interaction term among anticoagulant therapy, JAAM DIC score, and age. The lines indicate estimated log-transformed relative hazards, and the shaded areas represent 95\% confidence intervals. The solid blue line represents patients in the anticoagulant group, and the dotted red line represents those in the non-anticoagulant group. JAAM, Japanese Association for Acute Medicine; DIC, disseminated intravascular coagulation.

\section{Supplementary Files}

This is a list of supplementary files associated with this preprint. Click to download.

- SciRepSupplementaryTable.docx 